\title{
Avaliação do limite de estabilidade pelo Teste Alcance Funcional anterior em idosos
}

\section{Assessment of the limits of stability through the forward Functional Reach Test in older adults}

Matheus Augusto Braga Marques Vieira Rosa ${ }^{1}$, Arlete Maria Valente Coimbra², Adriana Ferreira do Nascimento $^{1}$, (Datalia Aquaroni Ricci ${ }^{1}$

\section{RESUMO}

O Teste Alcance Funcional avalia o limite de estabilidade, isto é, o quanto o indivíduo pode se movimentar sem alterar sua base de sustentação. Objetivo: Avaliar idosos pelo Teste Alcance Funcional (AF) e verificar os fatores associados ao seu desempenho. Métodos: Pesquisa observacional de corte transversal com análise secundária de dados coletados em estudo prévio. Amostra de idosos da comunidade ( $\geq 65$ anos), de Unidade de Saúde da Família, de ambos os sexos e com deambulação independente. Foram coletadas do banco as informações sociodemográficas, antropométricas, clínicas e de equilíbrio corporal (AF, Time Up and Go-TUG e Escala de Equilíbrio de Berg). O AF foi mensurado em uma única tentativa pelo deslocamento anterior do idoso, sendo classificado de forma numérica $(\mathrm{cm})$ e categórica pela Berg. Foi realizada estatística descritiva e inferencial (testes de correlação e associação) Resultados: Foram avaliados 96 idosos com média de 74,8 anos, AF de 22,5 $\pm 7,2 \mathrm{~cm}$ e 49\% conseguiram alcançar à frente mais que $25 \mathrm{~cm}$. Houve correlação entre o AF e variáveis sociodemográficas (idade), antropométricas (altura, peso e comprimento do pé), clínicas (força de preensão palmar e dor) e de equilíbrio (TUG e Berg). Idosos com faixa etária mais avançada, com doença endócrina, baixa acuidade visual, sedentários, com histórico de quedas, com queixas de dor e tontura apresentaram estatisticamente pior desempenho no AF. Conclusão: Idosos de Unidade de Saúde da Família apresentam AF levemente reduzido em relação à normalidade. Alguns fatores estão associados ao desempenho no AF e devem ser considerados na interpretação de seus resultados.

Palavras-chave: Equilíbrio Postural, Atenção Primária à Saúde, Idoso

\section{ABSTRACT}

The Functional Reach assesses the limits of stability, that is, how far the individual can move without changing the base of support. Objective: To evaluate the Forward Functional Reach Test (FR) in older adults and to verify the factors associated with the test performance. Method: Observational cross-sectional study with secondary analysis of data from a previous study. Sample of community-dwelling older adults ( $\geq 65$ years) from the Family Health Program, both sexes and independent for ambulation. Socio-demographic, anthropometric, clinical and balance control (FR, Time Up and Go-TUG and Berg Balance Scale) information were collected. The FR was measured in a single attempt by the anterior displacement of the subject classified numerically $(\mathrm{cm})$ and categorically according to the Berg Balance Scale. Descriptive and inferential statistical analysis (correlation and association tests) were performed. Results: 96 older adults were evaluated with mean age of 74.8 years, FR of $22.5 \pm 7.2 \mathrm{~cm}$ and $49 \%$ achieved more than $25 \mathrm{~cm}$. There was a correlation between FR and sociodemographic (age), anthropometric (height, weight and foot length), clinical (grip strength and pain) and balance (TUG and Berg Scale) data. Old people with more advanced age, with endocrine disease, low visual acuity, sedentary, with history of falls, with complaints of pain and dizziness presented statistically worse performance in FR. Conclusion: Older adults from the Family Health Program have a slightly reduced in FR compare to normative data from community-dwelling elderly. Some factors are associated with FR performance and should be considered when interpreting their results.

Keywords: Postural Balance, Primary Health Care, Aged 


\section{INTRODUÇÃO}

A bipedestação ou postura em pé é uma tarefa complexa, pois exige a manutenção constante do centro de gravidade dentro de sua base de sustentação. ${ }^{1} \mathrm{O}$ limite de estabilidade é considerado a área em que o corpo pode movimentar o centro de gravidade sem ter que alterar sua base de suporte. ${ }^{1,2} \mathrm{O}$ controle do limite de estabilidade envolve aspectos funcionais, anatômicos e biomecânicos do corpo. ${ }^{3} \mathrm{~A}$ avaliação do limite de estabilidade pode ser realizada por testes laboratoriais, como as posturografias; e por testes clínicos, como o Teste de Alcance Funcional.

$O$ teste Alcance Funcional Anterior (AF), conhecido internacionalmente como Forward Functional Reach Test, avalia a distância máxima que o indivíduo pode chegar à frente com o membro superior estendido mantendo a base de suporte fixa. ${ }^{2,4} \mathrm{O}$ teste fo criado em 1990, ${ }^{4}$ com o objetivo de avaliar de forma rápida, com baixo custo e com poucos materiais o limite de estabilidade. ${ }^{4,5} \mathrm{O}$ AF é considerado um teste preciso (coeficiente de variação de $2,5 \%$ ) e confiável (coeficiente de correlação intraclasse-ICC $=0,81$ ) na avaliação da população geral (21-87 anos). ${ }^{4}$ Para idosos da comunidade, o AF mostrou boa confiabilidade (ICC $=0,75-0,99) .{ }^{6}$

O AF é amplamente utilizado para avaliar o equilíbrio dinâmico em idosos. 2,5,7-9 O AF também está incorporado em outras avaliações de equilíbrio como a Escala de Equilíbrio de $\operatorname{Berg}^{10}$ e a Balance Evaluation Systems Test (BESTest). ${ }^{11} \mathrm{Na}$ Escala de Equilíbrio de Berg são considerados normais os valores do AF iguais ou superiores a $25 \mathrm{~cm},{ }^{11}$ e na BESTest valores superiores a $32 \mathrm{~cm} \cdot{ }^{11}$

Recente revisão sistemática com meta-análise ${ }^{12}$ revelou valores normativos do AF para idosos da comunidade de $26,6 \mathrm{~cm}$ (IC 95\%: 25,1 a $28,0 \mathrm{~cm}$ ) e de $15,4 \mathrm{~cm}$ (IC 95\%: 13,4 a $17,4 \mathrm{~cm}$ ) para idosos com alta demanda de cuidados em saúde (ex. institucionalizados e hospitalizados). Em outra meta-análise que incluiu idosos com condições específicas de saúde o valor normativo do AF foi de $27,2 \mathrm{~cm}$ (IC 95\%: 25,5 a 28,9 cm). ${ }^{13}$

A instabilidade postural e consequentemente as quedas são mais frequentes nos idosos devido às alterações fisiológicas do envelhecimento, à presença de doenças crônicas e ao uso de vários medicamentos. $^{1}$ Algumas pesquisas revelaram que a idade avançada, ${ }^{2,4,14}$ baixa estatura, 2,4,14 redução da força muscular ${ }^{2,4,14}$ e o medo de cair ${ }^{2,4,14}$ estavam relacionados com pior desempenho no AF.

Idosos do sexo masculino com valores inferiores à $25 \mathrm{~cm}$ no $\mathrm{AF}$ apresentaram risco aumentado de oito a duas vezes para quedas recorrentes, porém o risco não permaneceu para a ocorrência de uma única queda. ${ }^{5}$ Assim, ainda não existe consenso para uso do AF como medida padrão para avaliação do risco de quedas em idosos. ${ }^{12}$

Acredita-se que idosos inseridos em serviços de atenção primária procurem assistência para prevenção de agravos ou por algum problema de saúde e isso pode ocasionar valores diferenciados do AF.

Os dados do $\mathrm{AF}$ no contexto da atenção primária à saúde podem ser utilizados como parâmetros para evolução do tratamento da instabilidade postural e risco de quedas.

\section{OBJETIVO}

Avaliar o desempenho de idosos da atenção primária em saúde quanto ao equilíbrio dinâmico pelo AF e verificar os fatores que podem estar associados ao desempenho no teste.

\section{MÉTODOS}

Trata-se de pesquisa observacional de corte transversal realizada por meio de análise secundária de dados de estudo realizado no ano de 2006. A pesquisa intitulada "Análise comparativa entre idosos da comunidade em relação ao histórico de quedas quanto ao equilíbrio estático e dinâmico" 15 gerou um banco de dados com informações sobre idosos da comunidade acompanhados em Unidade de Saúde da Família (USF). Esta pesquisa está de acordo com os princípios éticos contidos na Declaração de Helsinki (2000) e com as Resoluções no 466/2012 e no 510/2016, tendo sido aprovada pelo Comitê de Ética em Pesquisa sob o número (766/2005).

Inicialmente foi realizada triagem para seleção dos participantes através de um banco de pesquisa populacional sobre envelhecimento e a Estratégia de Saúde da Família no município de Amparo (SP). ${ }^{16}$

$\mathrm{Na}$ triagem foi identificada maior concentração de possíveis voluntários para este estudo em uma única USF localizada próxima à região central da cidade. Os idosos desta unidade foram recrutados por meio dos agentes comunitários para avaliação dos critérios para participação no estudo.

Os critérios de inclusão dos participantes foram: idade igual ou superior a 65 anos, ambos os sexos e deambulação independente (sem dispositivos auxiliares para a marcha). Foram excluídos os idosos com limitações físicas e sensoriais decorrentes de problemas de saúde não controlados ou em estágio avançado ou com sequela incapacitante que pudessem interferir em testes de equilíbrio (ex. incapacidade de compreensão, acuidade visual e/ou auditiva gravemente diminuídas, amputação e/ou uso de próteses para membros inferiores e /ou superiores, sequelas de acidente vascular encefálico, doença de Parkinson, doença coronariana grave, fraturas de membros inferiores e /ou coluna após 65 anos, labirintopatia incapacitante, neuropatia e deformidade nos pés).

Os idosos elegíveis foram convidados a participar da pesquisa de forma voluntária, e aqueles que se dispuseram a participar assinaram o Termo de Consentimento Livre e Esclarecido. Todos os idosos foram avaliados em um único momento durante visita agendada pelos pesquisadores na USF. O protocolo completo de avaliação da pesquisa teve aproximadamente uma hora e meia de duração.

Para este estudo de análise secundária foram extraídos do banco de dados somente as informações referentes ao teste AF e após consulta na literatura foram selecionadas as possíveis variáveis com relevância/ interferência no teste..$^{1,12}$

As informações sociodemográficas extraídas foram: idade (anos), faixa etária (65 a 69 anos; 70 a 74 anos; 75 a 79 anos e acima de 80 anos) e sexo. Da avaliação clínica foram selecionados os dados de comorbidades (número total e classes de doenças relacionadas com o equilíbrio corporal-doenças visuais, auditivas/vestibulares, do sistema nervoso, osteomusculares/ou tecido conjuntivo, endócrinas nutricionais e/ou metabólicas, e alterações mentais e/ou comportamentais), número de medicamentos, queixa de tontura e dor, prática de atividade física, força muscular, acuidade visual e quedas.

As queixas de tontura e dor musculoesquelética, foram referentes à presença ( $\mathrm{sim} / \mathrm{não}$ ) de sintomas persistentes nos últimos seis meses, que caracterizaram a cronicidade da sintomatologia. Para a queixa de dor ainda foram coletados dados referentes à intensidade pela Escala Visual Analógica $(e m \mathrm{~cm})$ e sua localização (membros superiores, membros inferiores ou coluna) nas últimas 24 horas.

$\mathrm{O}$ idoso foi considerado praticante regular de atividade física ou sedentário conforme sua participação em atividades físicas em seu tempo livre por três ou mais vezes durante a semana, por mais de trinta minutos nas duas últimas semanas. ${ }^{17}$

A acuidade visual foi avaliada por meio da tabela direcional $E$ de Snellen, com o idoso sentado a uma distância de 5 metros da tabela que estava posicionada ao nível dos olhos. O teste foi realizado permitindo ao idoso o uso de lentes corretivas, caso fossem de uso habitual, e em ambos os olhos. A acuidade foi classificada em normal $(\geq 0,7)$, próxima do normal $(0,6$ a 0,3$)$, moderada $(0,25$ a 0,12$)$, e grave $(\leq 0,1) \cdot{ }^{18}$

A força muscular foi medida através da preensão palmar por meio de dinamômetro manual em $\mathrm{kg}$. Para o teste, o idoso foi posicionado sentado com braço aduzido e paralelo ao tronco, cotovelo fletido a 90 graus, antebraço e punho em posição neutra sendo orientado a apertar com a mão dominante o aparelho. $O$ valor final da força de preensão foi da média de três medidas. ${ }^{19}$ 
Em relação ao histórico de queda, foi perguntado ao idoso sobre o histórico de queda nos últimos 12 meses. A queda foi definida como um evento não intencional que tem como resultado a mudança de posição do indivíduo para um nível inferior em relação a sua posição inicial, sem perda da consciência ou resultante de força externa como um acidente inevitável. ${ }^{20}$

Ainda em relação as quedas, foram realizadas perguntas sobre o medo de sofrer quedas, tendência à queda, e a direção da queda no caso de histórico positivo. Para a avaliação do risco de queda pelo AF utilizou-se a nota de corte proposta por Duncan et al. ${ }^{5}$ de $25 \mathrm{~cm}$.

Os dados antropométricos de peso $(\mathrm{kg})$ e altura $(\mathrm{m})$ foram aferidos por balança de consultório e calculado o Índice de Massa Corpórea (IMC). O comprimento do pé $(\mathrm{cm})$ foi avaliado com o participante pisando em um papel, no qual foram marcadas as regiões anterior do maior artelho e posterior do calcanhar, sendo medida a distância entre estas marcas por fita métrica.

Para verificar a relação do AF com outras medidas de mobilidade e equilíbrio corporal foram aplicados o Teste Time Up and Go (TUG) e a Escala de Equilíbrio de Berg. O TUG avalia a mobilidade do idoso por meio do tempo despendido (segundos) em levantar-se de uma cadeira, caminhar três metros em linha reta, retornar à cadeira e sentar-se. ${ }^{21}$ Foi solicitado ao idoso que realizasse o teste calçado e em velocidade de marcha habitual em uma única tentativa. ${ }^{21}$

A Escala de Equilíbrio de Berg é considerada uma avaliação do equilíbrio funcional por ser composta de 14 atividades de vida diária que requerem controle do equilíbrio corporal para a execução. ${ }^{10} \mathrm{Cada}$ atividade pode ser pontuada de zero (incapaz de realizar) a quatro pontos (realiza de forma segura com bom equilíbrio). Sua pontuação total varia de 0 a 56 pontos, quanto maior a pontuação melhor o equilíbrio funcional.

$\mathrm{O}$ Teste AF foi mensurado por meio do item oito da Escala de Equilíbrio de Berg ${ }^{10}$ que consiste em "Alcançar a frente com braço estendido permanecendo em pé". Para sua realização foi fixada fita métrica na parede para medir o deslocamento anterior do idoso em $\mathrm{cm}$. O indivíduo estava descalço, e com um dos braços com o cotovelo estendido e flexão de ombro de 90 graus, mão esticada e aberta.

O participante deveria alcançar à frente a maior distância possível restringindo a movimentação do quadril e sem tirar o apoio dos calcanhares do solo. $O$ deslocamento anterior foi medido uma única vez considerando como marca final a falange distal do terceiro dedo. ${ }^{10}$

Para esta pesquisa foi utilizado o valor total de deslocamento $(\mathrm{cm})$ e as categorias propostas pela Escala de Equilíbrio de Berg para este item que são:

- alcança a frente mais que $25 \mathrm{~cm}$ com segurança.

- alcança a frente mais que $12,5 \mathrm{~cm}$ com segurança.

- alcança a frente mais que $5 \mathrm{~cm}$ com segurança.

- alcança a frente, mais necessita de supervisão.

- perde equilíbrio na tentativa, ou necessita de apoio externo.

A análise dos dados está apresentada de forma descritiva por média e desvio padrão, e por frequência absoluta e relativa (porcentagem). Os dados foram analisados quanto à normalidade pelo teste de Kolmogorov Smirnov. Os dados numéricos do AF apresentaram distribuição normal, porém as demais variáveis quantitativas não obtiveram normalidade. Assim, para as análises de associação do $\mathrm{AF}$ numérico com as variáveis categóricas foram utilizados os testes paramétricos T-Student e ANOVA.

Para a correlação entre o AF numérico e as demais variáveis quantitativas foi utilizado o teste de Spearman. A correlação fo considerada como "irrelevante" ( $0>r \leq 0.25)$, "fraca" (0.25>r $\leq 0.50)$, "moderada" $(0.50>r \leq 0.75)$ ou "forte" $(0.75>r \leq 1.00) .22$

Em relação ao evento queda foi feita análise da sensibilidade e especificidade considerando a nota de corte de $25 \mathrm{~cm}$. As análises foram feitas por meio do Programa computacional SPSS versão 17.0 com nível de significância de $5 \%$ para os testes estatísticos.

\section{RESULTADOS}

A amostra foi composta por 96 idosos igualmente selecionados quanto ao sexo, com 48 homens (50\%) e 48 mulheres (50\%), e média de idade de $74,8 \pm 6,9$ anos. A média do $A F$ foi de $22,5 \pm 7,2 \mathrm{~cm}$, com $49 \%$ dos idosos $(n=47)$ alcançando à frente com segurança mais de $25 \mathrm{~cm}$ (Figura 1). A caracterização da amostra está detalhada na Tabela 1.

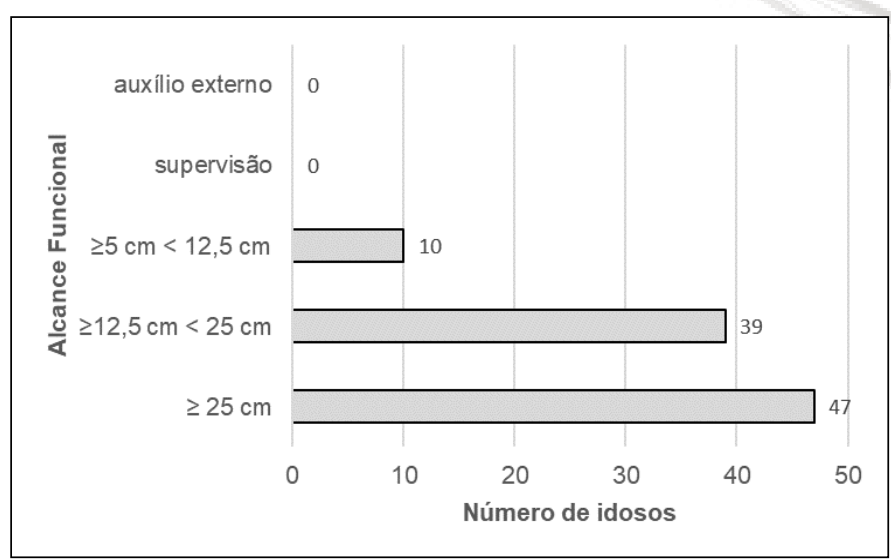

Figura 1. Percentual de idosos no Teste Alcance Funcional Anterior pela categorização da Escala de Equilíbrio de $\operatorname{Berg}(\mathrm{N}=96)$

Tabela 1. Associação entre dados demográficos e clínicos com o Teste de Alcance Funcional Anterior em idosos $(\mathrm{N}=96)$

\begin{tabular}{|c|c|c|c|}
\hline Variável & $\mathrm{n}(\%)$ & $\begin{array}{c}\text { Valor AF } \\
\text { Média } \pm \text { DP }\end{array}$ & p-valor \\
\hline \multicolumn{4}{|l|}{ Sexo } \\
\hline Feminino & $48(50,0)$ & $21,7 \pm 6,8$ & \multirow{2}{*}{0,31} \\
\hline Masculino & $48(50,0)$ & $23,2 \pm 7,7$ & \\
\hline \multicolumn{4}{|l|}{ Faixa Etária } \\
\hline $65-69$ & $24(25,0)$ & $25,7 \pm 8,4$ & \multirow{4}{*}{$0,01 *$} \\
\hline 70-74 & $24(25,0)$ & $21,9 \pm 6,0$ & \\
\hline 75-79 & $24(25,0)$ & $23,2 \pm 7,6$ & \\
\hline 80-mais & $24(25,0)$ & $19,2 \pm 5,6$ & \\
\hline \multicolumn{4}{|l|}{ Doença do olho e anexo } \\
\hline Sim & $20(20,8)$ & $22,0 \pm 6,7$ & \multirow{2}{*}{0,74} \\
\hline Não & & $22,6 \pm 7,5$ & \\
\hline \multicolumn{4}{|l|}{ Doença do ouvido } \\
\hline Sim & $10(10,4)$ & $21,3 \pm 9,5$ & \multirow{2}{*}{0,57} \\
\hline Não & & $22,6 \pm 7,0$ & \\
\hline \multicolumn{4}{|l|}{ Doença do sistema nervoso } \\
\hline Sim & $8(8,3)$ & $21,1 \pm 10,3$ & \multirow{2}{*}{0,57} \\
\hline Não & & $22,6 \pm 7,0$ & \\
\hline \multicolumn{4}{|c|}{ Doença do sistema osteomuscular } \\
\hline Sim & $44(45,8)$ & $22,6 \pm 6,3$ & \multirow{2}{*}{0,92} \\
\hline Não & & $22,4 \pm 8,0$ & \\
\hline \multicolumn{4}{|c|}{ Doença endócrina e/ou metabólicas } \\
\hline Sim & $30(31,3)$ & $21,5 \pm 7,4$ & \multirow[b]{2}{*}{0,04} \\
\hline Não & & $24,7 \pm 6,6$ & \\
\hline \multicolumn{4}{|l|}{ Transtornos Mentais } \\
\hline Sim & $6(6,3)$ & $22,2 \pm 8,2$ & \multirow{2}{*}{0,92} \\
\hline Não & & $22,5 \pm 7,2$ & \\
\hline \multicolumn{4}{|l|}{ Acuidade Visual } \\
\hline Visão Normal & $39(40,6)$ & $25,3 \pm 7,1$ & \multirow{3}{*}{$0,00 * *$} \\
\hline Visão Próxima do normal & $40(41,7)$ & $22,0 \pm 6,7$ & \\
\hline Baixa visão moderada & $17(17,7)$ & $17,3 \pm 5,9$ & \\
\hline \multicolumn{4}{|l|}{ Prática de atividade física } \\
\hline Sim & $23(24,0)$ & $25,8 \pm 6,8$ & \multirow{2}{*}{0,01} \\
\hline Não & & $21,4 \pm 7,1$ & \\
\hline \multicolumn{4}{|l|}{ Tontura } \\
\hline Sim & $34(35,4)$ & $19,5 \pm 7,5$ & \multirow{2}{*}{0,00} \\
\hline Não & & $24,1 \pm 6,6$ & \\
\hline \multicolumn{4}{|l|}{ Dor } \\
\hline $\operatorname{Sim}$ & $59(61,5)$ & $21,3 \pm 7,6$ & \multirow{2}{*}{0,04} \\
\hline Não & & $24,3 \pm 6,4$ & \\
\hline \multicolumn{4}{|l|}{ Dor em membros inferiores } \\
\hline Sim & $35(36,5)$ & $20,9 \pm 7,2$ & \multirow{2}{*}{0,10} \\
\hline Não & & $23,4 \pm 7,1$ & \\
\hline
\end{tabular}


(Continuação)

\begin{tabular}{|c|c|c|c|}
\hline \multicolumn{4}{|c|}{ Dor em membros superiores } \\
\hline $\begin{array}{l}\text { Sim } \\
\text { Não }\end{array}$ & $13(15,5)$ & $\begin{array}{l}19,4 \pm 5,7 \\
23,0 \pm 7,4\end{array}$ & 0,09 \\
\hline \multicolumn{4}{|l|}{ Dor em coluna } \\
\hline $\begin{array}{l}\text { Sim } \\
\text { Não }\end{array}$ & & $\begin{array}{l}22,0 \pm 7,9 \\
22,8 \pm 6,9\end{array}$ & 0,56 \\
\hline \multicolumn{4}{|l|}{ Histórico de queda } \\
\hline Nenhuma queda & $32(33,3)$ & $24,6 \pm 6,8$ & \multirow{2}{*}{0,04} \\
\hline Quedas & $64(66,7)$ & $21,4 \pm 7,3$ & \\
\hline \multicolumn{4}{|l|}{ Medo de queda } \\
\hline Sim & $57(59,4)$ & $21,6 \pm 6,9$ & \multirow{2}{*}{0,13} \\
\hline Não & & $23,8 \pm 7,6$ & \\
\hline \multicolumn{4}{|l|}{ Quase quedas } \\
\hline Sim & $37(38,5)$ & $21,8 \pm 8,0$ & \multirow{2}{*}{0,49} \\
\hline Não & & $22,9 \pm 6,8$ & \\
\hline \multicolumn{4}{|l|}{ Direção da queda } \\
\hline Lateral direita & $6(6,3)$ & $20,7 \pm 6,9$ & \multirow{4}{*}{0,65} \\
\hline Lateral esquerda & $9(9,4)$ & $20,6 \pm 4,8$ & \\
\hline Retropulsão & $12(12,5)$ & $19,5 \pm 8,0$ & \\
\hline Propulsão & $37(38,5)$ & $22,4 \pm 7,7$ & \\
\hline
\end{tabular}

* Pos-hoc: diferença significativa entre as faixas etárias de $65-69$ anos com 80 anos e mais ( $p=0,01)$

** Pos-hoc: diferença significativa entre visão normal e baixa visão moderada $(p<0,001)$

Foi encontrada associação entre o $\mathrm{AF}$ e algumas variáveis demográficas e clínicas (Tabela 1). Os indivíduos com idade avançada (80 anos e mais), doença endócrina, baixa visão moderada, sedentários, com queixa de dor e tontura, e histórico de quedas apresentaram valores mais baixos significativamente no AF. As demais variáveis estudadas não apresentaram associação com o AF.

Houve correlação entre o AF e a idade (anos), altura (m), peso (kg), comprimento do pé $(\mathrm{cm})$, intensidade da dor, força de preensão palmar, TUG e Escala de Berg. Foram encontradas correlações moderadas para as medidas de mobilidade e equilíbrio, enquanto que para as demais variáveis as correlações foram consideradas fracas. Não foi verificada correlação com comorbidades, polifarmácia e IMC (Tabela 2).

Tabela 2. Correlação entre dados demográficos, antropométricos e clínicos com o Teste de Alcance Funcional Anterior de idosos ( $N=96$ )

\begin{tabular}{lccc}
\hline \multicolumn{1}{c}{ Variável } & $\begin{array}{c}\text { Média } \pm \\
\text { DP }\end{array}$ & $\boldsymbol{p}$-valor & $\begin{array}{c}\text { Correlação Spearman } \\
\text { AF }(\mathbf{r})\end{array}$ \\
\hline Idade (anos) & $74,8 \pm 6,9$ & $\mathbf{0 , 0 0 1}$ & $-0,33$ \\
Comorbidades & $3,0 \pm 1,5$ & 0,49 & $-0,71$ \\
Número de Medicamentos & $3,2 \pm 2,0$ & 0,28 & $-0,11$ \\
Altura (m) & $1,5 \pm 0,9$ & $\mathbf{0 , 0 0 3}$ & 0,30 \\
Peso (Kg) & $68,5 \pm 13,2$ & $\mathbf{0 , 0 1}$ & 0,25 \\
IMC & $27,5 \pm 4,5$ & 0,48 & 0,07 \\
Comprimento do Pé (cm) & $25,5 \pm 2,9$ & $\mathbf{0 , 0 0 5}$ & 0,28 \\
Força de preensão palmar & $27,4 \pm 9,1$ & $\mathbf{0 , 0 0 0}$ & 0,37 \\
EVA Dor (cm) & $3,2 \pm 3,0$ & $\mathbf{0 , 0 1}$ & $-0,25$ \\
TUG (seg) & $13,4 \pm 4,1$ & $\mathbf{0 , 0 0 0}$ & $-0,51$ \\
Escala Equilíbrio de Berg & $50,2 \pm 4,4$ & $\mathbf{0 , 0 0 0}$ & 0,58 \\
\hline AF: Alcance Funcional; $E V A=$ Eacala Visual Analógica; IMC= Índice de Massa
\end{tabular}

AF: Alcance Funcional; EVA= Eacala Visual Analógica; IMC= Índice de Massa Corporal; $T U G=$ Time Up and Go

Em relação ao histórico de quedas (sem queda versus $\geq 1$ queda), ao utilizar a nota de corte de $25 \mathrm{~cm}$ foi verificado que 35 idosos com quedas (verdadeiro positivo) e 14 idosos do grupo sem queda (falso positivo) não alcançaram valores iguais ou superiores a este. Desta forma, para o ponto de corte de $25 \mathrm{~cm}$ a sensibilidade foi de $54 \%$, a especificidade de $56 \%$, o valor preditivo positivo de $71 \%$ e o valor preditivo negativo de $38 \%$.

\section{DISCUSSÃO}

Este estudo revelou que idosos atendidos na atenção primária pela Estratégia de Saúde da Família apresentam valores médios do AF inferiores aos dados normativos preconizados pelos autores que desenvolveram 0 teste (homens $=33,4 \pm 3,9 \mathrm{~cm}$ e mulheres $=$ $26,5 \pm 8,9 \mathrm{~cm}),{ }^{4}$ ao de estudo com idosos brasileiros (homens=
$29,7 \pm 2,8 \mathrm{~cm}$ e mulheres $=27,1 \pm 2,8 \mathrm{~cm}) 2$ e ao de meta-análise ${ }^{12}$ com idosos da comunidade de 26,6 cm (IC95\%: 25,1 a 28,0).

Deste modo, por estarem recebendo algum tipo de tratamento em USF estes idosos parecem ter o $\mathrm{AF}$ levemente diminuído em relação à idosos da comunidade em geral. Contudo, a categoria da Escala de Equilíbrio de Berg ${ }^{10}$ com maior número de idosos foi a de alcance superior a $25 \mathrm{~cm}$ sendo este valor considerado dentro da normalidade.

Assim, os idosos de USF apresentam resultados intermediários entre aqueles da comunidade e de outros ambientes terapêuticos, como hospital e atenção domiciliar, de $15,4 \mathrm{~cm}(95 \% \mathrm{Cl}: 13,4 ; 17,4),{ }^{12}$ que requerem maiores cuidados. $O$ reconhecimento precoce de idosos que estejam fora do padrão de normalidade do AF pode evitar futuro declínio do equilíbrio corporal e a necessidade de intervenção em níveis mais especializados de assistência.

Nesta pesquisa foi verificada ausência de diferença dos valores do AF entre homens e mulheres. Esse achado confirma o resultado obtido pelo estudo original ${ }^{4}$ e por outras pesquisas. ${ }^{12,23,24}$ Talvez a diferença encontrada nos valores do $\mathrm{AF}$ em relação ao sexo em outros estudos esteja relacionada aos aspectos antropométricos e idade dos sujeitos. ${ }^{12,25}$ Com relação a antropometria, este estudo apresentou correlação do AF com a altura e comprimento dos pés.

Outros estudos reforçam a influência das variáveis altura ${ }^{2,4}$ e tamanho do pé4 no desempenho do teste. Deste modo, os profissionais devem considerar ajustes no AF conforme as características antropométricas de seu paciente.

A idade avançada é o principal fator relacionado às alterações em testes de equilíbrio. ${ }^{1}$ Assim, como no presente estudo, diversas pesquisas encontram valores reduzidos no $\mathrm{AF}$ nas faixas etárias mais avançadas. 2,4,12 Em meta-análise com estudos que analisaram a influência da idade no AF foi verificado que após os 65 anos há um decréscimo progressivo no $A F$ de $0,53 \mathrm{~cm}$ por ano. ${ }^{12} \mathrm{O}$ profissional pode fazer correções no AF de seu paciente individual conforme a idade.

O número de comorbidades e a presença de doenças, exceto pela doença endócrina/metabólica, não tiveram impacto no resultado do AF. Entretanto, as queixas dos idosos (déficit visual, dor e tontura) estiveram associadas a pior desempenho no teste. Em estudo com idosos em atendimento fisioterapêutico, as queixas subjetivas (saúde global, audição, tontura, visão e dor em membros inferiores) e a presença de diabetes (doença endócrina/metabólica) apresentaram associação com maior dependência funcional. ${ }^{26}$

Ainda corroborando com nossos resultados, Almeida et al. ${ }^{23}$ sugerem que o AF apresenta-se diminuído entre idosos que referem perda visual. Isso mostra a importância de realizar a avaliação global do idoso e ouvir as queixas do paciente. Tudo indica que mais do que ter um diagnóstico, os sintomas e sua gravidade é que irão determinar o impacto na vida do indivíduo.

Vale notar que as variáveis que tiveram associação-correlação (tontura, déficit visual, dor, doença endócrina/metabólica, força de preensão) com o AF estão relacionados aos sistemas sensoriais (vestibular, visual, proprioceptivo) e ao sistema efetor responsáveis pelo equilíbrio corporal. É fundamental que os sistemas sensoriais e o sistema efetor estejam em bom funcionamento ${ }^{1,3}$ para garantir a estabilidade corporal.

Estes fatores podem estar relacionados com os resultados levemente abaixo da normalidade para os testes de equilíbrio avaliados nesta pesquisa, o AF, o TUG e a Escala de Equilíbrio de Berg. Além das correlações moderadas entre o AF e o TUG ( $r=-0.4427)$ e a Escala de Equilíbrio de Berg $(r=0.4727)$ que corroboram com resultados de outro estudo. ${ }^{27}$

Isso mostra que o limite de estabilidade, avaliado pelo AF, é um componente complexo do equilíbrio dinâmico e que requer inúmeros fatores para sua perfeita execução. Deste modo, as alterações em qualquer um dos componentes do controle postural pode levar a valores do $A F$ reduzidos.

As alterações e doenças nos sistemas responsáveis pelo controle postural podem causar perda de equilíbrio em idosos durante 
atividades associadas à movimentação do centro de gravidade ${ }^{27}$ e predispor o idoso as quedas. O histórico de quedas foi outro fator associado ao teste, isto é, os idosos caidores apresentaram pior desempenho no AF. Outras pesquisas de corte transversal também encontraram associação entre o $\mathrm{AF}$ e a presença de quedas. ${ }^{27-29}$

Entretanto, estudos prospectivos ${ }^{30-31}$ não encontraram relação entre o risco de queda e o AF. Importante relatar que este estudo por ser de corte transversal apresenta potencial viés de memória para predizer quedas. Além disso, este estudo demonstrou que o AF apresenta baixos valores de sensibilidade e especificidade para a nota de corte de $25 \mathrm{~cm}$. Deste modo, é necessário estudar novos pontos de corte para predição de risco de quedas em idosos acompanhados em USF.

Com o envelhecimento normalmente há um decréscimo do nível de atividade física. Nesta pesquisa a maioria dos idosos era sedentária. Resultado similar foi apresentado em estudo japonês, ${ }^{24}$ no qual o nível de atividade física influenciou no desempenho do AF. Com o envelhecimento, a atividade física torna-se fator importante para a manutenção do equilíbrio corporal, força muscular e flexibilidade; e consequentemente reflete em melhor desempenho no AF. A atividade física pode ser facilmente incorporada nas propostas terapêuticas para idosos na atenção primária à saúde.

O presente estudo apresenta limitações por ser uma análise secundária utilizando método de aplicação do $\mathrm{AF}$ diferente ao original ${ }^{4}$ e histórico retrospectivo de quedas. No método de aplicação do AF foi utilizada uma única tentativa, que difere do proposto por Duncan et al. ${ }^{4}$ com média de três tentativas e dois testes para adaptação.

Contudo, outro estudo com idosos demonstrou que o número de tentativas no AF não influencia em seu resultado, isto é, o uso de uma ou duas tentativas, ou a média de duas tentativas não é estatisticamente diferente dos resultados da média de três tentativas. $^{32}$ Novos estudos que testem o AF com diferentes metodologias de aplicação (posicionamento das mãos, base de sustentação) e que façam o acompanhamento prospectivo das quedas podem auxiliar na melhor interpretação do teste.

Por ser uma medida rápida e confiável, profissionais de USF podem incluir o AF na avaliação abrangente do idoso e utilizar seus resultados em comparação com os valores expostos por esta pesquisa para inferir sobre seu paciente individual, e propor estratégias de prevenção de instabilidade corporal.

\section{CONCLUSÃO}

Este estudo mostrou que a maioria dos idosos atendidos em USF apresentam teste de Alcance Funcional Anterior próximos da normalidade. E ainda, que alguns fatores, especialmente antropométricos e queixas subjetivas, estão associados ao desempenho do idoso no AF e devem ser considerados na interpretação de seus resultados no processo de reabilitação.

\section{REFERÊNCIAS}

1. Alexander NB. Postural control in older adults. J Am Geriatr Soc. 1994;42(1):93-108

2. Silveira KRM, Matas SLA, Perracini MR. Avaliação do desempenho dos testes functional reach e lateral reach em amostra populacional brasileira. Rev Bras Fisioter. 2006;10(4):381-6.

3. Ku PX, Abu Osman NA, Wan Abas WAB. The limits of stability and muscle activity in middle-aged adults during static and dynamic stance. J Biomech. 2016;49(16):3943-8. DOI: https://doi.org/10.1016/j.jbiomech.2016.11.006

4. Duncan PW, Weiner DK, Chandler J, Studenski S. Functional reach: a new clinical measure of balance. J Gerontol. 1990;45(6):M192-7.

5. Duncan PW, Studenski S, Chandler J, Prescott B. Functional reach: predictive validity in a sample of elderly male veterans. J Gerontol. 1992;47(3):M93-8.
6. Langley FA, Mackintosh SF. Functional balance assessment of older community dwelling adults: a systematic review of the literature. IJAHSP. 2007;5(4):1-11.

7. Uritani $D$, Fukumoto $T$, Matsumoto $D$, Shima $M$. The relationship between toe grip strength and dynamic balance or functional mobility among community-dwelling japanese older adults: a cross-sectional study. J Aging Phys Act. 2016;24(3):459-64. DOI https://doi.org/10.1123/japa.2015-0123

8. Tantisuwat A, Chamonchant D, Boonyong S. Multi-directional reach test: an investigation of the limits of stability of people aged between 20-79 Years. J Phys Ther Sci. 2014;26(6):877-80. DOI https://doi.org/10.1589/jpts.26.877

9. Balasubramanian C. Can gait and balance assessments accurately discriminate fallers from nonfallers in high-functioning community-dwelling older adults? Arch Phys Med Rehabil. 2014;95(10):e94-95.

DOI: https://doi.org/10.1016/j.apmr.2014.07.316

10. Berg KO, Wood-Dauphinee SL, Williams JI, Maki B. Measuring balance in the elderly: validation of an instrument. Can J Public Health. 1992;83 Suppl 2:S7-11.

11. Horak FB, Wrisley DM, Frank J. The Balance Evaluation Systems Test (BESTest) to differentiate balance deficits. Phys Ther. 2009;89(5):484-98. DOI: https://doi.org/10.2522/ptj.20080071

12. Rosa MV, Perracini MR, Ricci NA. Usefulness, assessment and normative data of the Functional Reach Test in older adults: A systematic review and meta-analysis. Arch Gerontol Geriatr. 2019;81:149-170. https://doi.org/10.1016/j.archger.2018.11.015

13. Bohannon RW, Wolfson LI, White WB. Functional reach of older adults: normative reference values based on new and published data. Physiotherapy. 2017;103(4):387-391. DOI https://doi.org/10.1016/j.physio.2017.03.006

14. Gabbard C, Cordova A. Association between imagined and actual functional reach (FR): a comparison of young and older adults. Arch Gerontol Geriatr. 2013;56(3):487-91. DOI https://doi.org/10.1016/j.archger.2012.12.008

15. Ricci NA, Gonçalves DFF, Coimbra IB, Coimbra AMV. Fatores associados ao histórico de quedas de idosos assistidos pelo Programa de Saúde da Família. Saúde Soc. 2010;19(4):898-909. DOI: http://dx.doi.org/10.1590/S0104-12902010000400016

16. Coimbra AM, Ricci NA, Coimbra IB, Costallat LT. Falls in the elderly of the Family Health Program. Arch Gerontol Geriatr. 2010;51(3):317-22.

DOI: https://doi.org/10.1016/j.archger.2010.01.010

17. Yusuf HR, Croft JB, Giles WH, Anda RF, Casper ML, Caspersen CJ, et al. Leisure-time physical activity among older adults. United States, 1990. Arch Intern Med. 1996;156(12):1321-6.

18. WHO. International Classification of Diseases, 9 th Revision (ICD9). Geneva: WHO; 1977.

19. Livingstone T BD, Carroll M. Grip Track Commander - user's manual. Utah: Jtech; 1997.

20. Tinetti ME, Speechley M, Ginter SF. Risk factors for falls among elderly persons living in the community. N Engl J Med. 1988;319(26):1701-7.

21. Podsiadlo D, Richardson S. The timed "Up \& Go": a test of basic functional mobility for frail elderly persons. J Am Geriatr Soc. 1991;39(2):142-8. DOI: https://doi.org/10.1111/j.15325415.1991.tb01616.x

22. Vieira S. Introdução à bioestatística. 4 ed. Rio de Janeiro: Elsevier; 2008.

23. Almeida ST, Soldera CLC, Carli GA, Gomes I, Resende TL. Análise de fatores extrínsecos e intrínsecos que predispõem a quedas em idosos. Rev Assoc Med Bras. 2012;58(4):427-33. DOI: http://dx.doi.org/10.1590/S0104-42302012000400012 
24. Sakamoto R, Okumiya K, Ishine M, Wada T, Fujisawa M, Imai H, et al. Predictors of difficulty in carrying out basic activities of daily living among the old-old: A 2-year community-based cohort study. Geriatr Gerontol Int. 2016;16(2):214-22. DOI: https://doi.org/10.1111/ggi.12462

25. de Waroquier-Leroy L, Bleuse S, Serafi R, Watelain E, Pardessus V, et al. The Functional Reach Test: strategies, performance and the influence of age. Ann Phys Rehabil Med. 2014;57(6-7):452-64. DOI: https://doi.org/10.1016/j.rehab.2014.03.003

26. Rossi AL, Pereira VS, Driusso P, Rebelatto JR, Ricci NA. Profile of the elderly in physical therapy and its relation to functional disability. Braz J Phys Ther. 2013;17(1):77-85. DOI: http://dx.doi.org/10.1590/S1413-35552012005000060

27. Newton RA. Validity of the multi-directional reach test: a practical measure for limits of stability in older adults. J Gerontol A Biol Sci Med Sci. 2001;56(4):M248-52. DOI: https://doi.org/10.1093/gerona/56.4.M248
28. GaiJ, Gomes L, Nóbrega OT, Rodrigues MP. Fatores associados a quedas em mulheres idosas residentes na comunidade. Rev Assoc Med Bras. 2010;56(3):327-332. DOI: http://dx.doi.org/10.1590/S0104-42302010000300019

29. Norris B, Medley A. The effect of context and age on functional reach performance in healthy adults aged 21 to 94 years. J Geriatr Phys Ther. 2011;34(2):82-7. DOI: https://doi.org/10.1519/jpt.0b013e31820aac1

30. Morita M, Takamura N, Kusano Y, Abe Y, Moji K, Takemoto T, et al. Relationship between falls and physical performance measures among community-dwelling elderly women in Japan. Aging Clin Exp Res. 2005;17(3):211-6.

31. Fujimoto A, Hori $H$, Tamura $T$, Hirai $T$, Umemura $T$, Iguchi $F$, et al. Relationships between estimation errors and falls in healthy aged dwellers. Gerontology. 2015;61(2):109-15. DOI: https://doi.org/10.1159/000363571

32. Billek-Sawhney B, Gay J. The Functional Reach Test are 3 trials necessary? Top Geriatr Rehabil. 2005;21(2):144-8. 